\title{
The emerging science of body weight regulation and its impact on obesity treatment
}

\author{
Judith Korner ${ }^{1}$ and Louis J. Aronne ${ }^{2}$ \\ ${ }^{1}$ Columbia University College of Physicians and Surgeons, New York, New York, USA \\ ${ }^{2}$ Weill Medical College of Cornell University, New York, New York, USA \\ J. Clin. Invest. 111:565-570 (2003). doi:10.1172/JCI200317953.
}

Obesity is a complex disorder characterized by the accumulation of excess adipose tissue. While obesity has long been considered a behavioral disorder, discovery of the hormone leptin in 1994 catalyzed the field of obesity research by demonstrating the existence of an afferent humoral signal from adipose tissue to the central nervous system. Current evidence suggests that once adipose tissue accumulates, a system of overlapping neuroendocrine systems prevents it from diminishing. This counter-regulatory mechanism, which has probably evolved as protection against starvation and fetal or neonatal wastage, causes changes in appetite and metabolism that make volitional weight loss difficult to achieve. Obesity is defined in terms of BMI, calculated as weight $(\mathrm{kg}) /[\text { height }(\mathrm{m})]^{2}$. Although a continuous variable, BMI has been categorized based on epidemiologic data to denote the relative risk of developing comorbid conditions. A BMI less than 25 is considered to be normal, 25-29.9 is overweight, and greater than or equal to 30, obese. Data from the 1999 National Health Nutritional and Exercise survey demonstrated that $34 \%$ of adults in the United States were overweight, and $30.8 \%$ obese, resulting in a total of $64.8 \%$ above normal weight. The prevalence of overweight and obesity in children was $13 \%$, a doubling since 1980 , while adolescents have experienced a tripling in prevalence since then.

The Science in Medicine series is supported in part by a generous grant from the Doris Duke Charitable Foundation.

Address correspondence to: Judith Korner, Columbia University College of Physicians and Surgeons, 630 West 168th Street, Black Building, Room 905, New York, New York 10032, USA. Phone: (212) 305-3725; Fax: (212) 305-2282;

E-mail: jk181@columbia.edu.

Conflict of interest: Louis J. Aronne acts as a consultant, speaker, or advisor or receives research support from the following: Abbott Laboratories, Amgen, Bristol-Myers Squibb Company, OrthoMcNeil Pharmaceuticals, Otsuka Pharmaceuticals, Regeneron Pharmaceuticals, Roche Pharmaceuticals, Sanofi-Synthelabo Inc., and TAP Pharmaceutical Products Inc.

Nonstandard abbreviations used: diabetes mellitus (DM); janus kinase-signal transducers and activators of transcription (JAK-STAT); neuropeptide Y (NPY); melanin-concentrating hormone $(\mathrm{MCH})$; glucagon-like peptide-1 (GLP-1); $\alpha$-melanocytestimulating hormone $(\alpha-\mathrm{MSH})$; proopiomelanocortin (POMC); agouti-related protein (AGRP); peptide $\mathrm{YY}_{3-36}$ (PYY); Food and Drug Administration (FDA).
Being overweight or obese substantially increases the risk of morbidity from a number of conditions, including type 2 diabetes mellitus (DM), hypertension, dyslipidemia, coronary heart disease, congestive heart failure, stroke, gallbladder disease, hepatic steatosis, osteoarthritis, sleep apnea, and endometrial, breast, prostate, and colon cancers. An increase in all-cause mortality is also associated with higher body weights. Adipose tissue is an active endocrine organ that produces free fatty acids and hormones, such as IL-6, TNF- $\alpha$, plasminogen activation inhibitor-1, angiotensinogen, and others, directly related to the insulin resistance, hyperlipidemia, inflammation, thrombosis, and hypertension that characterize obesity. Visceral fat appears to be the greatest contributor to these effects, probably because of its location in the portal circulation draining to the liver.

\section{Regulation of energy homeostasis}

The discovery of leptin and other genes responsible for obesity in rodents has had a considerable impact on our understanding of body weight regulation. Leptin (derived from Greek leptos, meaning thin) is a hormone that is produced by fat cells and circulates at levels proportional to body fat content. Leptin crosses the bloodbrain barrier to bind to its receptor in the hypothalamus, thereby activating signals that inhibit food intake and increase energy expenditure. When leptin is given to leptin-deficient mice, obesity and metabolic abnormalities such as hyperglycemia, hyperinsulinemia, and hypercortisolemia are reversed. However, the initial hypothesis that human obesity results from a deficiency in leptin has not been upheld. In fact, most obese humans have high circulating concentrations of leptin, and only a few individuals with severe obesity have been identified either with congenital leptin deficiency or a mutation in the gene encoding the leptin receptor (Table 1). In early clinical trials, high doses of subcutaneously-administered leptin, resulting in greater than 20 -fold increases in circulating leptin concentrations, were often associated with reactions at the injection site and had only a modest effect in reducing body weight. Therefore, it has been suggested that obese persons are leptin resistant. If indeed a defect in the leptin pathway gives rise to human obesity, it may be due to a postreceptor defect in the transduction of leptin signaling 


\begin{tabular}{|c|c|c|}
\hline Gene variant & Mode of inheritance & Phenotypes in addition to obesity \\
\hline Leptin & Homozygote; loss of function & $\begin{array}{l}\text { Hypogonadotropic hypogonadism, possible } \\
\text { perturbations in growth hormone and thyroid axes }\end{array}$ \\
\hline Leptin & Heterozygote; loss of function & None \\
\hline Leptin receptor & Homozygote; loss of function & $\begin{array}{l}\text { Hypogonadotropic hypogonadism, } \\
\text { growth retardation, central hypothyroidism }\end{array}$ \\
\hline Proopiomelanocortin & $\begin{array}{l}\text { Homozygote and compound heterozygote; } \\
\text { loss of function }\end{array}$ & Altered pigmentation, adrenal insufficiency \\
\hline $\begin{array}{l}\text { Proopiomelanocortin: mutation } \\
\text { of dibasic processing site }\end{array}$ & Heterozygote; partial loss of function & None \\
\hline Prohormone convertase 1 & Compound heterozygote; loss of function & $\begin{array}{l}\text { Hypogonadotropic hypogonadism, } \\
\text { adrenal insufficiency, hyperproinsulinemia }\end{array}$ \\
\hline Melanocortin-4 receptor & Homozygote and heterozygote; loss of function & $\begin{array}{l}\text { Increased growth velocity, } \\
\text { increased bone mineral density in children }\end{array}$ \\
\hline
\end{tabular}

through the janus kinase-signal transducer and activator of transcription (JAK-STAT) pathway. The etiology of central leptin resistance may involve a suppressor of the cytokine-signaling family SOCS-3, which is induced by leptin and prevents activation of the JAK-STAT pathway (1). From an evolutionary perspective, the physiological function of leptin is probably as a metabolic switch that serves to preserve body fat during times of famine, rather than an antiobesity agent that suppresses body fat during times of food excess (2).

Leptin is known to affect the gene expression and synthetic pathway of both anorectic (appetite-suppressing) and orexigenic (appetite-stimulating) substances (Figure 1). Neuropeptide Y (NPY) is a hypothalamic orexigenic peptide produced in the arcuate nucleus, which increases food intake and decreases energy expenditure. The expression of NPY mRNA is inhibited by leptin. In the lateral hypothalamus, melanin-concentrating hormone $(\mathrm{MCH})$, an orexigenic peptide, is increased with fasting and leptin deficiency. Disruption of the $M C H$ gene or administration of an $\mathrm{MCH} 1$ receptor antagonist results in hypophagia and leanness in rodents $(3,4)$. Glucagon-like peptide-1 (GLP-1) and neurotensin are peptides that inhibit the ability of $\mathrm{MCH}$ to induce eating. Leptin also downregulates endocannabinoids that act as cannabinoid receptors in the hypothalamus and stimulate food intake (5). Conversely, leptin stimulates the expression of genes encoding anorexigenic peptides. $\alpha$-Melanocyte-stimulating hormone $(\alpha-\mathrm{MSH})$, a peptide derived from proopiomelanocortin (POMC), and cocaine- and amphetamine-regulated transcript are hypothalamic peptides expressed in the same subset of neurons within the arcuate nucleus of the hypothalamus. These peptides are positively regulated by leptin and produce anorexia.

The melanocortin system is under intensive investigation because of evidence in both rodents and humans of its control of energy homeostasis (6). There are five different receptors for $\alpha-\mathrm{MSH}$, two of which (MC3R and MC4R) are primarily expressed in the brain. The highest $M C 3 R$ expression level is in the hypothalamus and limbic system, whereas MC4R
mRNA is expressed in virtually all major brain regions. Some of the metabolic effects resulting from stimulation of MC4R are decreased food intake and an increase in energy expenditure through stimulation of thyrotropin-releasing hormone and activation of the sympathetic nervous system. Targeted deletion of the MC4R gene produces obesity, hyperphagia, hyperinsulinemia, and reduced energy expenditure. Targeted deletion of $M C 3 R$ results in increased adiposity due to decreased energy expenditure. The central melanocortin system also regulates insulin release and peripheral insulin responsiveness independent of its effects on feeding behavior and adiposity.

Although common obesity is likely to be a polygenetic disorder, monogenetic causes, especially within the melanocortin pathway, have been described (Table 1). Mutations in $M C 4 R$ are found in approximately $1-5 \%$ of humans with a BMI over 40 and are particularly associated with severe early-onset obesity. In addition, there have been reports of rare mutations in the POMC gene and the enzyme that processes the POMC protein to $\alpha$-MSH that are associated with severe childhood obesity. Further evidence for the importance of the melanocortin pathway stems from the identification of agoutirelated protein (AGRP). AGRP is a hypothalamic peptide that stimulates food intake in the rat through antagonism of the interaction of $\alpha-\mathrm{MSH}$ at MC4R. Expression of the AGRP gene is suppressed by leptin.

Gastrointestinal tissues also relay information to the brain about energy balance through neural and endocrine pathways. Insulin and its receptor have traditionally been studied for their role in glucose homeostasis. There is now mounting evidence that the central actions of insulin parallel that of leptin; central administration of insulin or insulin mimetics reduce food intake and body weight (7), whereas impairment of hypothalamic insulin receptors causes hyperphagia and insulin resistance (8). Another peptide, ghrelin, produced by the stomach, intestine, pituitary, and possibly hypothalamus, was originally described as an inducer of growth hormone release, but more recently its function as an orexigenic peptide has been elucidated (9). 
Intracerebroventricular injection of ghrelin stimulates feeding in the rat and increases body weight through stimulation of NPY and A GRP neurons. Intravenous injection of ghrelin in humans induces subjective hunger and food intake presumably by interaction with neurons in the arcuate nucleus of the hypothalamus that are accessible to peripheral hormones. The preprandial rise and postprandial fall in plasma ghrelin levels and correlation with hunger indicate that ghrelin may play a role in meal initiation in humans. In contrast to leptin and insulin, which are released in proportion to body fat stores and have long-term effects on energy homeostasis, ghrelin triggers short-term effects related to meals. Circulating ghrelin concentrations are decreased in obese humans, but current reports are conflicting as to whether food intake suppresses ghrelin levels in these individuals $(10,11)$.

Another gut hormone, peptide $\mathrm{YY}_{3-36}$ (PYY), is an agonist of the NPY Y2 receptor expressed on NPY neurons in the arcuate nucleus, which is released postprandially in proportion to the caloric content of a meal. Injection of PYY decreases NPY and increases POMC neuronal activity, and reduces food intake in rodents and humans (12). Cholecystokinin, which is released in response to dietary fat, enhances nutrient absorption by slowing gastric emptying and stimulating gallbladder contraction, and inhibits food intake during the meal via the afferent vagal system. GLP-1 inhibits gastrointestinal motility, hunger, and food intake. Secretion of gastric inhibitory polypeptide, a duodenal hormone, does not affect food intake, but promotes obesity through efficient storage of nutrients as fat (13).

Intracellular metabolites also regulate energy metabolism and may signal the availability of fuel to metabolite-sensitive hypothalamic neurons. Interference with central pathways involved in the synthesis of malonyl-
CoA or fatty acids with either genetic knockouts of acetyl-CoA carboxylase (14) or fatty acid synthase inhibitors such as cerulenin or C75 $(15,16)$ have been shown to decrease body fat. It has also been shown that an adipocyte-derived hormone, Acrp30 (also known as AdipoQ or adiponectin), increases fatty acid oxidation in muscle and liver and may regulate fat accumulation without significantly affecting food intake (17). Many other molecules, including other peptides, neurotransmitters, cytokines, steroid hormones, and enzymes, such as $11 \beta$-hydroxysteroid dehydrogenase, involved in steroid hormone metabolism, affect energy homeostasis but are beyond the scope of this review.

\section{Energetics of body weight regulation}

The high frequency of relapse and limited weight loss attainable with nonsurgical therapies are among the frustrations experienced by the obese patient. Investigation of the energetics of weight loss and weight gain demonstrates that maintenance of a reduced body weight is associated with declines in energy expenditure that are greater than can be accounted for by reductions in metabolic mass (18). The weightreduced state is also characterized by decreased circulating triiodothyronine and thyroxine concentrations, and reduced sympathetic and increased parasympathetic nervous tone. These changes persist for months to years in weight-reduced subjects. Administration of leptin to fasting rodents blunts the starvationinduced changes in gonadal, adrenal, and thyroid axes $(19,20)$ and ameliorates the increase in concentrations of mRNAs encoding hypothalamic orexigenic peptides (21). Leptin administration also attenuates the hyperphagia and reduced energy expenditure associated with sibutramine-induced weight loss in rats. Some of these metabolic, neuroendocrine, and

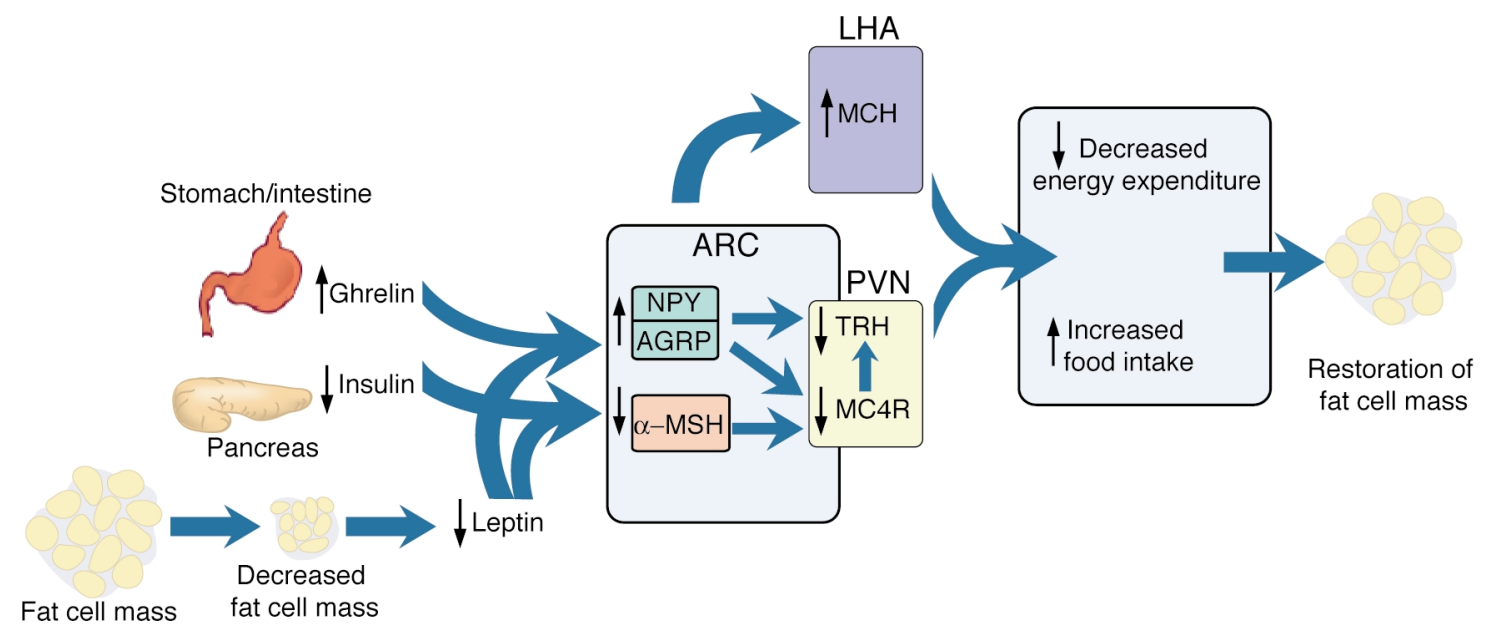

\section{Figure 1}

Interaction of some hormonal and neural pathways that regulate food intake and body fat mass. A decrease in fat cell mass is sensed in the arcuate nucleus (ARC) of the hypothalamus by a decrease in leptin and insulin concentrations, causing suppression of anorexigenic signals, such as $\alpha$-MSH, and stimulation of orexigenic signals such as AGRP and NPY. Neurons from the arcuate nucleus project to the paraventricular nucleus (PVN) and lateral hypothalamic area (LHA) to decrease TRH and increase MCH synthesis. The decrease in $\alpha-\mathrm{MSH}$ also decreases TRH biosynthesis and release. The gastrointestinal hormone, ghrelin, modulates these pathways through activation of NPY/AGRP neurons. The net balance of these signals results in an increase in food intake and a decrease in energy expenditure that ultimately aims to restore fat cell mass. 
Table 2

Weight loss drugs approved for long-term use (modified from ref. 24)

Drug

Sibutramine (Meridia)

Orlistat (Xenical)
Action

Norepinephrine, dopamine, and serotonin reuptake inhibitor Inhibits pancreatic lipase, decreases fat absorption
Adverse effects

Increase in heart rate and blood pressure Decrease in absorption of fat-soluble vitamins; soft stools and anal leakage behavioral phenotypes, which also characterize leptindeficient rodents and humans, are reversed by lowdose leptin administered to weight-reduced humans at doses that restore circulating leptin to the concentration preceding weight loss (22).

These findings support the concept that decreased circulating leptin concentration accounts for the integrated changes in behavior, neuroendocrine status, and energy expenditure that characterize the weightreduced state, and is partially responsible for the frequent regain of lost weight in weight-reduced subjects. The weight-reduced state is perceived by leptin-responsive pathways in the central nervous system as a leptindeficient state (2).

\section{Obesity treatment}

The primary target for treatment should be those individuals at medical risk because of their weight. This includes overweight and obese patients with a BMI greater than 25, particularly in the presence of comorbidities, an increased waist circumference (greater than $88 \mathrm{~cm}$ for women or $102 \mathrm{~cm}$ for men), or a family history of obesity. A realistic treatment goal is usually loss of $5-10 \%$ of initial body weight over a 6-12 month period followed by long-term maintenance of reduced weight. Most cardiovascular risk factors are improved even at this level of modest weight reduction because of the predominant loss of visceral fat leading to a disproportionate decrease in the risk of developing complications. The recent Diabetes Prevention Program, for example, found that a mean weight loss of $5.6 \mathrm{~kg}$ achieved by diet and exercise reduced the risk of progression from impaired glucose tolerance to type 2 diabetes by $58 \%$ over a 4 -year period (23). For more detail, a guide to obesity treatment published by the National Institutes of Health and the North American Association for the Study of Obesity is available online (24).

\section{Nonpharmacological treatments for obesity}

Nonpharmacological treatments for obesity include behavior therapy, exercise, and calorie-restricted diets. The goal of behavior therapy is to overcome barriers to compliance with a diet and physical activity regimen. Physical activity increases energy expenditure and is a key component of any weight maintenance program, counteracting the reduction in total energy expenditure that occurs with weight loss. In general, patients should build up to a 30 -to 45 -minute exercise regimen, daily if possible, of the greatest intensity exercise that is safe for their own level of fitness. Improvement in cardiovascular risk is observed through regular exercise even if no weight is lost (25).
In order to induce weight loss a calorie deficit must be created. The optimal macronutrient composition of a weight-reducing diet is controversial. The use of lowfat, high-carbohydrate diets has been challenged by small trials suggesting that hyperinsulinemic patients may benefit from a lower glycemic diet. It has been proposed that the rapid absorption of glucose from the high-glycemic index meal results in relatively high insulin and low glucagon concentrations, which induce a reactive hypoglycemia that stimulates hunger and favors storage of fat. Fat-modified foods lower total fat intake, but are only effective in achieving weight loss if they are also low in calories.

\section{Pharmacological treatments currently available}

Individuals enrolled in controlled trials of the two prescription drugs, sibutramine and orlistat, currently approved by the Food and Drug Administration (FDA) for long-term use in the United States, demonstrate a placebo-subtracted weight loss of 4-6\% over a 6-month period, depending upon the intensity of the diet, exercise, and behavioral program administered (Table 2). Like diabetes or hypertension, obesity must be treated chronically. In most cases, medication, once started, must be used at least intermittently for weight loss to be maintained.

Sibutramine (Meridia; Abbott Laboratories, Abbot Park, Illinois, USA) is a reuptake inhibitor of serotonin, norepinephrine, and dopamine and was initially developed as an antidepressant. In animals, sibutramine administration is associated with reduced expression of NPY mRNA. Sibutramine has been shown to decrease food intake and may increase thermogenesis. The most common adverse events seen with sibutramine include constipation, dry mouth, and insomnia. An increase in heart rate and blood pressure may occur in some individuals.

Orlistat (Xenical, Hoffman-LaRoche, Nutley, New Jersey, USA) is an inhibitor of gastrointestinal lipases that blocks $30 \%$ of dietary fat from being absorbed. Comorbidities improve with orlistat, although total and LDL cholesterol improve more than expected because of direct inhibition of fat absorption. Adjunctive orlistat administration over a 4-year period reduced the risk of progression to type $2 \mathrm{DM}$ in an at-risk population more than diet and exercise alone. Multivitamin supplementation is recommended because orlistat may also reduce the absorption of lipid-soluble vitamins.

Noradrenergic-releasing agents, such as the schedule IV drugs phentermine (Ionamin, Fastin, Adipex) and diethylpropion (Tenuate), induce more weight loss than placebo in short-term studies. Since large-scale, 
long-term studies have not been performed, these agents are approved only for short-term use.

\section{Surgery}

The most common surgical approach to treating obesity is the Roux-en-Y gastric bypass, in which a small gastric pouch prevents the patient from eating large quantities at a single meal and a gastrojejunostomy produces a dumping syndrome if the patient ingests high-carbohydrate foods. Suppression of ghrelin production by the excluded stomach may be another mechanism of weight loss. In the longest follow-up study of patients after bypass, Pories et al. reported $58 \%, 55 \%$, and $49 \%$ loss of excess weight (defined as the patient's weight minus the patient's estimated "ideal" body weight) at 5, 10, and 14 years postoperatively (26). Deficiencies of iron, calcium, thiamin, and vitamins A, $\mathrm{C}, \mathrm{D}$, and $\mathrm{B}_{12}$ have been observed, and long-term supplementation is recommended.

Restrictive procedures such as laparoscopic adjustablebanded gastroplasty are simpler, safer procedures than gastric bypass, but are not as effective. An inflatable device that encircles the stomach is inserted laparoscopically and forms a small pouch. Adjustment of the stoma is readily accomplished by inflating the device with saline via a subcutaneous port. Patients are unable to eat large volumes at a single meal, but they can ingest highcaloric density liquids without suffering any ill effects.

\section{Future therapeutic strategies}

The direct costs of obesity and obesity-related diseases represent about $6 \%$ of national health expenditures in the United States. The sum of annual direct and indirect costs is approximately $\$ 100$ billion. Of special concern is the rapidly increasing prevalence of childhood obesity, which has doubled to greater than $14 \%$ over the past thirty years. Without an effective environmental and public education initiative, the prevalence of this disease is expected to continue to rise among children and adults worldwide. Until successful preventive strategies are instituted, more effective treatments will be necessary.

A myriad of new targets for obesity treatment has arisen from the recent advancement in our understanding of the complex circuitry controlling energy homeostasis (Table 3). It appears that orexigenic signals are redundant as a safeguard for survival. Thus impairment of one signal, such as the knockout of AGRP, NPY, or ghrelin, does not produce any obvious metabolic disorder. Effective weight-loss therapy will most likely involve blockade of the action of more than one orexigenic factor to yield a rational combined therapy. For example, therapies that combine anorexigens with drugs that shift lipid partitioning toward oxidation and decrease insulin resistance may be more effective than either class used alone. Another goal of research in this field is to understand how short-term signals from the gastrointestinal tract integrate with longer-term signals of metabolic status within the central nervous system and influence decisions regarding meal termination and frequency. Knowledge of these processes may provide critical therapeutic targets.
Initially, the finding that circulating ghrelin concentrations are decreased in obese compared with lean humans suggested that the use of ghrelin antagonists in the treatment of common obesity may not be effective. However, ghrelin concentrations are decreased further after weight loss induced by gastric bypass surgery, as opposed to increasing after diet-induced weight loss, suggesting that ghrelin antagonists may ameliorate hunger and aid in the maintenance of weight loss. In addition, ghrelin antagonists may be particularly effective in the treatment of obesity in patients with PraderWilli syndrome, who have several-fold higher concentrations of ghrelin compared to equally obese controls. The effect of such antagonists on growth hormone secretion will need to be elucidated.

Several drugs are currently in preclinical or clinical trials. One drug, rimonabant, a cannabinoid receptor 1 antagonist, suppresses tonic endogenous activation of the orexigenic endocannabinoid system. Another, axokine, is an analog of ciliary neurotrophic factor, which appears to activate the central leptin pathway distal to the leptin receptor. Both drugs are now in phase III clinical trials. Other promising therapies include sustained-release bupropion, an antidepressant that is a dopamine and norepinephrine reuptake inhibitor, and the antiepileptic drug, topiramate. Topiramate has multiple pharmacologic effects, but it is unclear which of these contribute to reductions in energy intake and efficiency. Metformin, an FDA-approved medication for the treatment of type $2 \mathrm{DM}$, which inhibits hepatic glucose production and improves sensitivity to insulin, induces small weight loss and reduces the risk of progression from impaired glucose tolerance to type $2 \mathrm{DM}$ (23). Inhibitors of tyrosine phosphatase-IB, an enzyme that appears to be involved in the mechanism of leptin resistance, have shown promise in preclinical studies. Stimulation of thermogenesis may become possible with the development of potent, yet specific, $\beta_{3}$-adrenergic-receptor agonists. Interestingly, it has been shown

Table 3

Potential obesity treatments in development ${ }^{\mathrm{A}}$

Agonists

Ciliary neurotrophic factor or analogues

Glucagon-like peptide-1

Insulin mimetics and/or sensitizers

Leptin, leptin analogues and/or sensitizers

Melanocortin-4 receptor agonists

Dopaminergic, noradrenergic, serotinergic agents

Antagonists

Endocannabinoid receptor antagonists

Fatty acid synthesis inhibitors

Ghrelin antagonists

Melanin-concentrating hormone receptor antagonists

Neuropeptide $Y$ receptor antagonists

Tyrosine phosphatase-IB inhibitors

${ }^{A}$ Although other drugs are in development, this table is limited primarily to substances described in the text. 
that the anorexic action of $\mathrm{d}$-fenfluramine, a serotonergic agent that was withdrawn from the market due to adverse cardiopulmonary events, requires activation of central melanocortin pathways, indicating that $\mathrm{MSH}$ analogs or drugs that target downstream melanocortin pathways may be effective without incurring serotoninassociated side effects.

The evidence is overwhelming that obesity, once established, is a multiorgan endocrinopathy of body weight regulation. That medical treatment for this disorder will ultimately be more effective seems increasingly obvious. Clearly the time is ripe for the development of new weight-loss drugs. In the near future we should see therapies specifically aimed at elements within the weight control circuitry that will act with greater efficacy, perhaps when used in combination, but will not produce the untoward side effects associated with past treatments. At that time, the treatment of obesity could come to dominate the outpatient management of the many chronic diseases to which an increased body weight contributes.

\section{Acknowledgments}

The authors gratefully acknowledge the review of this manuscript by Sharon L. Wardlaw and Rudolph L. Leibel. This work was supported by NIH grant DK59316 (to J. Korner).

1. Bjørbæk, C., Elmquist, J.K., Frantz, J.D., Shoelson, S.E., and Flier, J.S. 1998. Identification of SOCS-3 as a potential mediator of central leptin resistance. Mol. Cell. 1:619-625.

2. Leibel, R.L. 2002. The role of leptin in the control of body weight. Nutr. Rev. 60:S15-S19.

3. Borowsky, B., et al. 2002. Antidepressant, anxiolytic and anorectic effects of a melanin-concentrating hormone-1 receptor antagonist. Nat. Med. 8:825-830.

4. Tritos, N.A., and Maratos-Flier, E. 1999. Two important systems in energy homeostasis: melanocortins and melanin-concentrating hormone. Neuropeptides. 33:339-349.

5. Di Marzo, V., et al. 2001. Leptin-regulated endocannabinoids are involved in maintaining food intake. Nature. 410:822-825.

6. Wardlaw, S.L. 2001. Clinical review 127: Obesity as a neuroendocrine disease: lessons to be learned from proopiomelanocortin and melanocortin receptor mutations in mice and men. J. Clin. Endocrinol. Metab. 86:1442-1446.

7. Air, E.L., et al. 2002. Small molecule insulin mimetics reduce food intake and body weight and prevent development of obesity. Nat. Med. 8:179-183.

8. Obici, S., Feng, Z., Karkanias, G., Baskin, D.G., and Rossetti, L. 2002. Decreasing hypothalamic insulin receptors causes hyperphagia and insulin resistance in rats. Nat. Neurosci. 5:566-572.

9. Nakazato, M., et al. 2001. A role for ghrelin in the central regulation of feeding. Nature. 409:194-198.

10. Cummings, D.E., et al. 2002. Plasma ghrelin levels after diet-induced weight loss or gastric bypass surgery. N. Engl. J. Med. 346:1623-1630.

11. English, P.J., Ghatei, M.A., Malik, I.A., Bloom, S.R., and Wilding, J.P. 2002. Food fails to suppress ghrelin levels in obese humans. J. Clin. Endocrinol. Metab. 87:2984-2987.

12. Batterham, R.L., et al. 2002. Gut hormone $\mathrm{PYY}_{(3-36)}$ physiologically inhibits food intake. Nature. 418:650-654.

13. Miyawaki, K., et al. 2002. Inhibition of gastric inhibitory polypeptide signaling prevents obesity. Nat. Med. 8:738-742.

14. Abu-Elheiga, L., Matzuk, M.M., Abo-Hashema, K.A., and Wakil, S.J. 2001. Continuous fatty acid oxidation and reduced fat storage in mice lacking acetyl-CoA carboxylase 2. Science. 291:2613-2616.

15. Loftus, T. M., et al. 2000. Reduced food intake and body weight in mice treated with fatty acid synthase inhibitors. Science. 288:2379-2381.

16. Makimura, H., et al. 2001. Cerulenin mimics effects of leptin on metabolic rate, food intake, and body weight independent of the melanocortin system, but unlike leptin, cerulenin fails to block neuroendocrine effects of fasting. Diabetes. 50:733-739.

17. Fruebis, J., et al. 2001. Proteolytic cleavage product of $30-\mathrm{kDa}$ adipocyte complement-related protein increases fatty acid oxidation in muscle and causes weight loss in mice. Proc. Natl. Acad. Sci. U. S. A. 98:2005-2010.

18. Leibel, R.L., Rosenbaum, M., and Hirsch, J. 1995. Changes in energy expenditure resulting from altered body weight. N. Engl. J. Med. 332:621-628

19. Ahima, R.S., et al. 1996. Role of leptin in the neuroendocrine response to fasting. Nature. 382:250-252.

20. Legradi, G., Emerson, C.H., Ahima, R.S., Flier, J.S., and Lechan, R.M. 1997. Leptin prevents fasting-induced suppression of prothyrotropinreleasing hormone messenger ribonucleic acid in neurons of the hypothalamic paraventricular nucleus. Endocrinology. 138:2569-2576.

21. Korner, J., et al. 2001. Leptin regulation of Agrp and Npy mRNA in the rat hypothalamus. J. Neuroendocrinol. 13:959-966.

22. Rosenbaum, M., Murphy, E.M., Heymsfield, S.B., Matthews, D.E., and Leibel, R.L. 2002. Low dose leptin administration reverses effects of sustained weight-reduction on energy expenditure and circulating concentrations of thyroid hormones. J. Clin. Endocrinol. Metab. 87:2391-2394.

23. Knowler, W.C., et al. 2002. Reduction in the incidence of type 2 diabetes with lifestyle intervention or metformin. N. Engl. J. Med. 346:393-403.

24. National Institutes of Health, National Heart Lung and Blood Institute, and North American Association for the Study of Obesity. 2000. The practical guide: identification, evaluation, and treatment of overweight and obesity in adults. NIH. Bethesda, Maryland, USA. Publication no. 00-4084.

25. Lee, C.D., Blair, S.N., and Jackson, A.S. 1999. Cardiorespiratory fitness, body composition, and all-cause and cardiovascular disease mortality in men. Am. J. Clin. Nutr. 69:373-380.

26. Pories, W.J., et al. 1995. Who would have thought it? An operation proves to be the most effective therapy for adult-onset diabetes mellitus. Ann. Surg. 222:339-350. 